\title{
The level of bilirubin and the risk of ischemic stroke: a systematic review and dose-response meta-analysis of real-world studies
}

\author{
Xiao Wang \\ Yang Zhou \\ Bengbu Medical College \\ Xiaofei Ye \\ Second Military Medical University \\ Fangchen Liu \\ Shanghai University of Traditional Chinese Medicine \\ Xi Zhu \\ Shanghai University of Traditional Chinese Medicine \\ Danhong Wu \\ Fifth People's Hospital of Shanghai Fudan University \\ Ping Zhong ( $\sim$ zphgl@163.com )
}

Shanghai Traditional Chinese and Western Medicine Hospital https://orcid.org/0000-0001-9786-9199

Research article

Keywords: Bilirubin; biomarker; risk factor; ischemic stroke; systematic review; dose-response meta-analysis

Posted Date: May 4th, 2020

DOI: https://doi.org/10.21203/rs.2.15743/v2

License: @) (1) This work is licensed under a Creative Commons Attribution 4.0 International License. Read Full License 


\section{Abstract}

Background: Bilirubin, a marker of hepatic and hematological diseases in clinical practice, is not only a waste end-product but also an antioxidant that may protect against diseases associated with oxidative stress. Numerous epidemiological studies have shown an inverse relationship between the serum total bilirubin (TBIL) level and the risk of ischemic stroke (IS). However, markedly elevated TBIL levels may exert neurotoxic effects. Based on this, we conducted a dose-response meta-analysis to quantify the relationship between blood TBIL and IS as well as between TBIL and all types of stroke (AS) in the physiological range of bilirubin.

Methods: PubMed, Embase, Web of Science, and Cochrane Central databases were searched up to March 2019. Additional studies were identified by reviewing references and contacting authors. Categorical and dose-response meta-analyses were performed to quantify the relationship between TBIL and IS. The primary outcome was ischemic stroke, and the secondary outcome was all types of stroke.

Results: Nine observational studies (seven publications) involving 110,032 participants and 3710 stroke cases were included for analysis. The average OR of IS for every $1 \mu \mathrm{mol} / \mathrm{L}$ increment in TBIL level was 0.978 (95\% Cl: $0.957-0.999)$. The summary OR of AS for every $1 \mu \mathrm{mol} / \mathrm{L}$ increment in TBIL level was 0.974 (95\% Cl: 0.956-0.992). Subgroup analysis based on gender showed a negative dose-response relationship between the circulating TBIL level and IS or AS in males, but not in females.

Conclusions: The present study found a negative dose-response relationship between the circulating TBIL level and the risk of IS or AS within physiologic range of serum TBIL in males. Moderately elevated blood TBIL levels might be associated with a diminished prevalence of IS. Every $1 \mu$ mol/L increment in serum TBIL level was associated with a $2.2 \%$ decrease in the risk of IS and a $2.6 \%$ decrease in the risk of AS. However, due to the limitations in the number of included studies and their quality, large-scaled prospective cohort studies are needed to confirm the conclusion of the current analysis.

Trial registration: This study was registered at PROSPERO (https://www.crd. york.ac.uk/PROSPERO/[CRD42017075988]).

\section{Background}

Stroke is the second-leading cause of death and the leading cause of long-term disability worldwide [1]. More than two thirds of stroke deaths worldwide occur in developing countries [2]. In China, stroke has been an enormous health issue as the most common cause of death in recent years [3]. Identifying and managing risk factors for stroke is highly important for the general population to prevent the occurrence of stroke. A number of serum biomarkers have been intensively studied in clinical trials of stroke and summarized according to their pathophysiological importance in stroke pathogenesis [4]. Among them, circulating total bilirubin (TBIL), a breakdown product of normal heme catabolism [5], is proved to be one of the strongest endogenous antioxidants in mammalian tissues [6]. Accumulating evidence has shown that bilirubin harbors anti-inflammatory [7], neuroprotective [8], and inhibiting platelet activation [9] effects. These properties entitle bilirubin to prevent or delay the formation of thrombi in ischemic stroke (IS), and to protect neurons from ischemic injury, suggesting that elevated bilirubin levels may be linked to a lower rate of morbidity and mortality of stroke patients. Recently, research has shifted to focus on its role in reducing the risk of developing strokes [10-18] though some of them showed only moderately positive or null effect [17, 18]. A large prospective study showed that every $1 \mu \mathrm{mol} / \mathrm{L}$ increment in bilirubin concentration was associated with a $2 \%$ reduction in the hazard ratio in a multivariate-adjusted model for IS and 1\% reduction in the hazard ratio for all types of stroke (AS). However, the participants are only Korean [19]. The National Health and Nutrition Examination Survey involving 13,214 adult participants reported that a $1.71 \mu \mathrm{mol} / \mathrm{L}$ increment in the bilirubin level was associated with a $9 \%$ reduction in the odds ratio for stroke. However, this is only a cross-sectional study [20]. Subsequently, a meta-analysis of 12 population-based prospective studies involving 9378 incidental cardiovascular disease (CVD) cases showed that every 1-SD increase in the TBIL level was associated with a 7\% reduction in the relative risk of CVD, but stroke was not the end point [11]. Zhong et al. reported an inverse relationship between the level of circulating TBIL and the risk of IS or AS in males through meta-analysis which included 11 observational studies involving 5,060 stroke cases among 131,450 subjects, but it is not a dose-response analysis [21].

At present, it is sufficiently clear that low serum bilirubin level was independently associated with an increased risk of incidental CVDs in the general population, but a markedly elevated TBIL level may exert neurotoxic effects [22,23] and the dose-response relationship between the circulating TBIL level and the risk of IS/AS has not been confirmed. We, therefore, aimed to explore the dose-response correlation between the level of blood TBIL and the incidence of IS within the physiological range of TBIL based on previously published literatures.

\section{Materials And Methods}

\section{Literature search}

Articles on blood TBIL levels and stroke in adults ( $\geq 18$ years), published up to March 2019, were systematically searched in databases of PubMed, Embase, Cochrane Central, and Web of Science by two authors (XW and YZ) independently. Detailed search strategies were shown in the Supplementary Material. Language was limited to English. In addition, references cited by relevant articles as well as those listed in published meta-analyses were manually searched to identify additional eligible studies. This study was registered at PROSPERO (https://www.crd.york.ac.uk/ PROSPERO/[CRD42017075988]), and the PRISMA and the MOOSE guidelines for meta-analyses of observational studies were followed [24, 25].

\section{Study selection}

The inclusion criteria were: 1) prospective cohort, case-cohort, or cross-sectional design; 2) studies investigating the relationship between serum TBIL and incidence of IS/AS; 3) estimates of adjusted RR (HR, OR) with 95\% Cls were included; 4) For the dose-response analysis, a quantitative measure of blood TBIL levels and the total number of cases and person-years or the number of events had to be provided. Abstracts, animal studies, in vitro studies, comments, 
letters, editorials, ecological studies, gray literature, unpublished results or information, and randomized controlled trials were excluded. The primary outcome of this study was ischemic stroke, and the secondary outcome was stroke (defined as all types of stroke).

\section{Data extraction}

From each included study, data including the last name of the first author, year of publication, country where the study was conducted, study name, type of study, follow-up period, sex, age, the number of stroke cases and the total number of study participants, bilirubin levels, adjusted ORs, RRs, or HRs of IS/AS (with corresponding $95 \% \mathrm{Cls}$ ), and variables adjusted for in the analysis were extracted. Two authors (XW and YZ) extracted the data into a standardized form, and a third author (PZ) checked them for accuracy.

\section{Quality assessment}

Biases within each individual study were evaluated by two independent reviewers (XW and YZ) using the validated ROBINS-I tool which provides a comprehensive and structured approach to assess non-randomized studies of interventions [26]. This tool covered domains of pre-intervention bias due to confounding factors and selection of participants, intervention bias in classifying interventions, and post-intervention bias due to deviation from intended interventions, missing data, measurement of outcomes, and selection of reported results. An overall judgment was reached after assessing each of the domains and classified as low, moderate, serious, critical risk of bias, or no information on which to base a judgment on the risk of bias. Disagreements were resolved by consensus or by a third-party adjudication $(X Y)$ as necessary.

\section{Statistical analysis}

Statistical analysis was performed using the Review Manager (version 5.1 for Windows, Cochrane Collaboration, Oxford, UK, 2011) and STATA 11.0 (Stata Corporation, Lakeway, Texas, USA). The ORs and associated $95 \% \mathrm{Cls}$ were considered as the effect sizes. Summary ORs and $95 \%$ Cls for the highest versus the lowest levels of serum TBIL and for the dose-response analysis were calculated. The average of the natural logarithm of ORs was estimated, and the OR from each study was weighed by the inverse of its variance. A previously described method [27] was used for the dose-response analysis and study specific slopes (linear trends) and 95\% confidence intervals from the natural logs of the odds ratios and confidence intervals across categories of blood bilirubin levels were computed. It required that the distribution of cases and person-years or non-cases, and the ORs with their variance estimates were known for at least three quantitative categories of bilirubin levels. If a study did not report the distribution of cases or person-years but the total number of cases or person-years, then the results were analyzed by quantiles (and could be approximated). The median or mean level of bilirubin in each category was assigned to the corresponding OR for each study. If the study reported the bilirubin level by ranges, the midpoint was estimated by calculating the average of the lower and upper bounds in each category. If the highest or lowest category was open ended, the length of the open-ended interval was assumed to be the same as that of the adjacent interval. Studies that reported results separately for men and women were deemed as two separate studies. The dose-response results in the forest plots were presented for every $1 \mu \mathrm{mol} / \mathrm{L}$ increment for IS or AS. A potential nonlinear dose-response relationship between circulating TBIL levels and all types of stroke was examined using fractional polynomial models [28]. A likelihood ratio test was used to assess the difference in nonlinearity between the nonlinear and linear models [29]. Generalized least squares for trend estimation and the restricted cubic spline regression model were applied in the doseresponse analysis.

Heterogeneity between studies was evaluated using $\chi^{2}$ test and $\mathrm{I}^{2}$ statistic, and between subgroups using meta-regression analyses. Heterogeneity was considered to be present when $P<0.1$. Random-effect models were used for all analyses. Publication bias was assessed using the Egger's test [30] and the funnel plot. Publication bias was indicated when $P$ was $<0.1$. Sensitivity analysis was carried out by excluding one study at a time to explore whether the result was driven by a large study or by a study with an extreme result. A two-tailed $P$ value less than 0.05 was considered statistically significant.

\section{Results}

The initial search yielded 422 studies from PubMed, 1471 from Embase, 10 from Cochrane Central, and 539 from Web of Science. After excluding of duplicates and irrelevant studies, 46 potentially relevant full-text publications were selected. 5 studies with categories of blood bilirubin levels $<3$, 5 studies with no original data, 1 case report, 1 conference literature, 1 letter, 4 review studies, 1 study with low quality and no clear data relationship, 6 studies with a comparison of unreasonable outcomes, and 15 irrelevant studies were excluded. A manual search of references of these studies did not yield any new eligible studies. Results of the study-selection process were shown in Fig. 1. Finally, nine observational studies (seven publications) [10, 13-16, 19, 20] were selected for analysis, including 2 prospective cohort studies [14, 19], 1 case-control study [10], and 4 cross-sectional studies [13, 15, 16, 20], involving 110,032 participants with 3710 stroke cases. The ranges of blood TBIL levels and general characteristics were shown in Table 1. Included studies were assessed for bias using the ROBINS-I tool. All studies in analysis had a moderate risk of bias (Supplementary Table $₫$ ). This meant that all included observational studies were judged to provide sound evidence as non-randomized studies but could not be considered comparable to well-performed randomized trials.

\section{Correlation between the level of circulating total bilirubin and ischemic stroke}

Five observational studies (4 publications) $[10,15,16,19]$ explored the association between TBIL and IS for the highest versus the lowest levels of serum TBIL and for the dose-response analysis, including two prospective cohort studies (1 publications) [19], 1 case-control study [10], and two cross-sectional studies $[15,16]$ involving 2312 ischemic stroke cases among 81,632 participants (Table 1). As Kimm 2009 separately reported results for men and women, we deemed them as two studies as mentioned previously.

Highest versus lowest bilirubin levels 
Five observational studies (4 publications) $[10,15,16,19]$ were included in the analysis of highest versus lowest levels of circulating TBIL and the risk of IS. There was mild heterogeneity between studies $\left(I^{2}=39.6 \%, P=0.157\right)$, and the random-effect model was used for this analysis. The summary odds ratio for

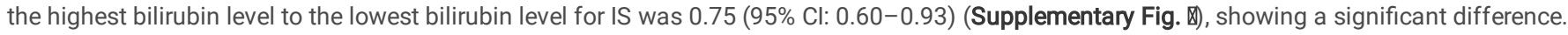

\section{Dose-response analysis}

In the dose-response meta-analysis investigating the relationship between TBIL and IS, the summary OR for every $1 \mu \mathrm{mol} / \mathrm{L}$ increment of the TBIL level was 0.978 (95\% Cl: 0.957-0.999, Fig. 2), with substantial heterogeneity $\left(1^{2}=84.3 \%, P<0.001\right.$, Supplementary Table $\left.\Downarrow\right)$. In the sensitivity analysis, excluding one study at a time did not show a particular study that could explain the results for IS. The summary OR ranged from 0.972 (95\% Cl: $0.949-0.996)$ when Zhou 2014 was excluded to 0.989 (95\% Cl: 0.982-0.996) when Kim 2017 was excluded (Supplementary Table \and Supplementary Fig. \). The publication bias was not evident as shown by the Egger's test $(P=0.620)$ (Supplementary Fig. $\mathbb{\text { Q }}$ ). A negative linear relationship between blood TBIL levels and the risk of IS was indicated $(P=0.392$, Fig. 3).

\section{Subgroup and meta-regression analyses}

Subgroup analysis of two prospective cohort studies (1 publication) [19] showed that the total OR for every $1 \mu$ mol/L increment of the TBIL level for IS was 0.988 (95\% Cl: 0.981-0.995) after adjusting for potential risk factors, showing a significant difference. In the two cross-sectional studies [15, 16], the summary OR for every $1 \mu \mathrm{mol} / \mathrm{L}$ increment of the TBIL level for IS was 0.968 (95\% Cl: 0.901-1.040) after adjusting for potential risk factors, showing no significant difference. There was mild heterogeneity in the cohort studies $\left(I^{2}=36.0 \%, P=0.211\right)$ and severe heterogeneity in the cross-sectional studies $\left(I^{2}=91.9 \%, P<\right.$ 0.001). In meta-regression analyses, no heterogeneity in types of stroke was observed between subgroups $(P=0.485)$ (shown in Supplementary Table $\rrbracket)$.

Subgroup analysis was performed based on gender difference. The total OR for every $1 \mu \mathrm{mol} / \mathrm{L}$ increment of TBIL for IS was 0.984 (95\% Cl: $0.974-0.993)$ for males, showing a significant difference; whereas the total OR for every $1 \mu \mathrm{mol} / \mathrm{L}$ increment of TBIL for IS was 0.992 (95\% Cl: 0.981-1.003) for females, showing no significant difference. A significant difference was observed in patients aged $<60$ years [0.988 ( $95 \%$ Cl: $0.981-0.995)]$, but not in those $\geq 60$ years [0.968 (95\% Cl: 0.901-1.040)]. In addition, subgroup analysis of studies with $\geq 2000$ subjects showed a significant difference [0.988 (95\% Cl: $0.981-0.995)$ ], which was not observed in those with <2000 subjects [0.968 (95\% Cl: 0.917-1.002)]. In subgroups further defined by the sample size, gender, and the average patient age, no significant heterogeneity between subgroups was observed in meta-regression analyses (All $P$ values $>0.1$ ) (shown in Supplementary Table $\llbracket$ ).

\section{Association of blood total bilinubin level with all types of stroke}

In nine observational studies (7 publications) $[10,13-16,19,20]$, the correlation between the level of bilirubin and the risk of AS from the highest to the lowest levels of serum TBIL and the dose-response analysis were investigated. There were 2 prospective cohort studies [14, 19], 1 case-control study [10], and 4 crosssectional studies [13, 15, 16,20], involving 110,032 participants including 3,710 stroke subjects. Both Kimm 2009 and Oda 2012 reported results for men and women. Hence, they were both deemed as two separate studies as mentioned previously (Table 1).

\section{Highest versus lowest bilirubin levels}

Nine observational studies (7 publications) were included in the analysis of highest versus lowest serum TBIL levels and the risk of AS. There was moderate heterogeneity $\left(I^{2}=45.3 \%, P=0.067\right)$ between these 9 studies, and the random-effect model was used. The summary odds ratio for the highest bilirubin to the lowest bilirubin for AS was 0.721 (95\% Cl: 0.606-0.858) (Supplementary Fig. 囚).

\section{Dose-response analysis}

Nine observational studies (7 publications) were included to investigate the exact dose-response relationship between TBIL and all types of stroke. The summary OR for every $1 \mu \mathrm{mol} / \mathrm{L}$ increment of the TBIL level for AS was 0.974 (95\% Cl: 0.956-0.992, Fig. 4), showing a significant difference. However, substantial heterogeneity was observed $\left(I^{2}=80.1 \%, P<0.001\right.$, Supplementary Table $\left.\rrbracket\right)$. No particular study explained this OR for all types of stroke in the sensitivity analysis. The summary OR ranged from 0.969 (95\% Cl: 0.951-0.988) when Kimm 2009 was excluded to 0.983 (95\% Cl: $0.969-0.996)$ when Zhou

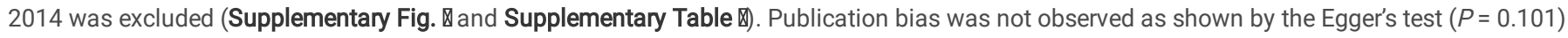
(Supplementary Fig. 『). A negative linear dose-response relationship was observed between the blood TBIL level and the incidence of AS $(P=0.315$, Supplementary Fig. (『).

\section{Subgroup and meta-regression analyses}

In 5 cross-sectional studies (4 publications) [13, 15, 16, 20], the total OR for AS was 0.965 (95\% Cl: $0.939-0.992$ ) after adjusting for potential risk factors, demonstrating a significant difference. However, in 3 cohort studies (2 publications) [14, 19], the total OR for every $1 \mu \mathrm{mol} / \mathrm{L}$ increment of the TBIL level for AS was 0.996 (95\% Cl: 0.988-1.004) after adjusting for potential risk factors, showing no significant difference. Heterogeneity was noted in 5 cross-sectional studies $\left(I^{2}=79.2 \%, P=0.001\right)$, but not in prospective cohort studies $\left(I^{2}=0 \%, P=0.424\right)$. Stratified analysis based on gender showed that the $0 \mathrm{R}$ was $0.982(95 \%$ Cl: $0.966-0.998)$ for males and 0.981 (95\% Cl: 0.951-1.012) for females, indicating a significant difference for males and no difference for females. Significant difference was also observed when the average age of patients was $<60$ years, but not when the average age was $\geq 60$ years. Moreover, subgroup analysis showed a significant difference in studies with a sample size $\geq 2000$ participants, but not in those with $<2000$ participants. In meta-regression

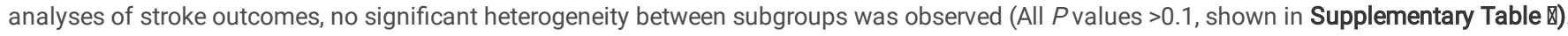

\section{Discussion}


Based on available data from observational studies, the present meta-analysis demonstrated a negative linear dose-response relationship between the serum TBIL level and the risk of IS or AS after adjusting for potential confounding factors. Every $1 \mu \mathrm{mol} / \mathrm{L}$ increment of the circulating TBIL level was associated with a $2.2 \%$ decreased risk of IS and a $2.6 \%$ decreased risk of AS.

Bilirubin, an end-product of heme catabolism, has been considered as a potentially toxic agent at high levels in the human body. However, it is now recognized as a natural antioxidant, especially against the oxidation of lipids. This property is believed to diminish plaque formation, thereby protecting against atherosclerosis and the subsequent macrovascular diseases [31]. A number of studies have demonstrated that serum TBIL was negatively correlated with the intima-media thickness of the carotid artery (IMT) [32-36]. In humans, a low (<7 $\mu \mathrm{mol} / \mathrm{L})$ TBIL concentration has been shown to be a risk factor for systemic diseases associated with increased oxidative stress, such as CVDs, diabetes, metabolic syndrome, certain cancers, autoimmune, and neuropsychiatric diseases [37]. As described in our recently published review, studies have demonstrated that the level of bilirubin may serve as a predictor of some vascular events, such as hypertension, coronary artery diseases, peripheral artery diseases, and so on [38]. Yang et al conducted a prospective cohort study in 90,532 participants who underwent health examination and found that the level of TBIL was an independent risk factor for new cerebral infarcts when its level was in the range of 1.0-9.7 $\mu \mathrm{mol} / \mathrm{L}$ [39]. Lin et al found that serum TBIL was an independent predictor of cardioembolic stroke [40]. Kurzepa et al indicated that the serum bilirubin level is a poor prognostic factor for ischemic stroke [41]. Previous studies also showed that a mild-to-moderate increase in the bilirubin level might be a promising strategy for preventing cardiovascular events [42-44]. The result of the present study coincided with previous reports that bilirubin might be an independent predictor of stroke incidence $[19,20]$. The negative association between serum TB and the incidence of IS may be explained by the mechanisms of bilirubin-mediated inhibition of lipid oxidation, immune reactions, inflammatory processes, cell migration and proliferation, as well as apoptosis $[22,23]$.

The key finding of our study is the negative dose-response relationship between the circulating TBIL level and the risk of IS or AS within physiologic range of serum TBIL in males. It is known that the level of bilirubin is one of the many risk factors of ischemic stroke, but whether manipulating the level of bilirubin can reduce the risk of developing stroke remains to be elucidated. It might be a potential target as reported by previous studies. It is known that statins can activate HO-1 and increase the production of bilirubin $[45,46]$. Reduction of glutathione - an antioxidant increases the production of bilirubin. Stress related hormones such as adrenocorticotropin also increases the level of bilirubin and decreases the risk of cardiogenic diseases [47, 48]. In Traditional Chinese Medicine, diverse decoctions, such as Angongniuhuang Wan for ischemic stroke, have high levels of bilirubin. Bilirubin might be the key component of these decoctions. But the apparent paradox between toxic and protective effects addresses again the double-edged sword effect of bilirubin. Clarification of the protective bilirubin cut-off level and intervention trials may answer the question whether bilirubin is a therapeutic target for stroke and CVDs [19].

The threshold is $0 \mu \mathrm{mol} / \mathrm{L}$ as shown in the dose-response curve (Fig. 3 and Supplementary Fig. ( ). Bilirubin, known as a potential toxic factor causing severe brain damage in newborns [49], is not only the end product of the heme catabolic pathway but also the most potent endogenous antioxidant [7]. It is naturally present in the blood and its level can not be 0 or lower. When its level is above 0 , the OR for ischemic stroke is less than 1 and this OR decreases as the level of bilirubin increases. This suggests that bilirubin is a protective factor. Previous studies have reported the threshold but it varies between studies. In a prospective cohort study consisting of 90,532 participants who underwent health examination, Yang et al found that the level of total serum bilirubin was an independent risk factor of new cerebral infarcts when its level was in the range of 1.0-9.7 $\mu \mathrm{mol} / \mathrm{L}$ [39]. A meta-analysis study indicated that 10.0 $\mu \mathrm{mol} / \mathrm{L}$ TBIL was a clear cut-off point for discriminating high from low cardiovascular risks [50].

Gilbert's syndrome (GS) is a benign hereditary disorder of bilirubin conjugation characterized by an isolated, elevated blood level of unconjugated bilirubin [51]. Normally (allowing for differing laboratory ranges), the blood TBIL level is $<17 \mu \mathrm{mol} / \mathrm{L}$ and clinical jaundice becomes apparent at levels $>40 \mu \mathrm{mol} / \mathrm{L}$. However, Gilbert's syndrome is considered a phenotypic effect rather than a serious disorder because of its profound beneficial effects. Studies have shown that GS could apparently protect against chronic inflammatory disorders including CVD [52], atherosclerosis [53], cancer and all-cause mortality [9], thus supporting the concept that mildly elevated unconjugated bilirubin could be protective against the development of future CVDs. Large prospective cohort studies are needed to explore the risk of IS/AS in phenotypic Gilbert syndrome defined as total bilirubin $>17 \mu \mathrm{mol} / \mathrm{L}$.

Serum TBIL consists of direct (DBIL) and indirect bilirubin (IBIL). Due to the lack of data in the literature regarding DBIL, IBIL and their relationship with stroke, we are unable to clarify which fraction of bilirubin is a risk factor of stroke in our meta-analysis and review because our result is based on previously published studies. However, this question has raised interest of a small number of researchers. A large cross-sectional study compared three types of bilirubin levels (TBIL, DBIL, and IBIL) in different subtypes of subcortical ischemic vascular disease (SIVD, included lacunar infarction (LI) and leukoaraiosis (LA)) [15]. It was observed that the decrease in bilirubin levels remained significant in both LA and LI subtypes when compared with controls. Moreover, bilirubin levels (TBIL, DBIL, and IBIL) were slightly lower in LA than LI in both males and females. Luo et al reported that serum levels of DBIL and TBIL were increased after AIS, which was linked to the severity of stroke. Other studies demonstrated the positive correlation between TBIL, DBIL and the NIHSS of ischemic stroke patients $[54,55]$. It was also found that TBIL, DBIL and IBIL levels were significantly associated with mortality in AIS patients [56]. Further large-scaled studies are needed to clarify this issue.

Stroke is categorized into two types: ischemic and hemorrhagic. The present meta-analysis found that the serum TBIL level was negatively correlated to the incidence of IS or AS. In our analysis, only one study investigated the relationship between serum TBIL and hemorrhagic stroke (HS) and did not find a significant relationship between them [19]. Pathophysiology of IS is different from that of HS. A stronger relationship between TBIL levels and the risk of IS than between TBIL levels and HS may support the antiatherogenic property of bilirubin as suggested in a previous study [20]. Moreover, IS is an etiologically heterogeneous disease and can also be categorized into different clinical subtypes. Lin et al conducted a study to explore the potential use of serum TBIL as a biomarker to differentiate cardioembolic stroke from other types of stroke [40]. In this study, 628 consecutive patients had IS whose etiology was classified into large artery atherosclerosis, cardioembolism, small vessel occlusion, other determined etiology, and undetermined etiology according to the TOAST criteria [57]. It was found that serum TBIL was an independent predictor of cardioembolic stroke. In a cross-sectional study, the association between TBIL and silent cerebral infarction ( $\mathrm{SCl}$ ) was evaluated among 2,865 subjects undergoing medical checkup. It was found that participants with a low level of TBIL had a 
higher prevalence of SCl. This suggests that TBIL may be a novel biomarker for SCI [58]. Further studies are needed to investigate the relationship between the serum TBIL level and the risk of different subtypes of IS.

In this study, a negative dose-response relationship was observed between the serum TBIL level and the incidence of IS or AS in males, but not in females. Vítek et al and Kunutsor et al also demonstrated an inverse relationship between circulating TBIL and carotid artery IMT [36] as well as CVDs [11] in males, respectively. The difference between men and women might be attributed to lifestyle factors, including tobacco, alcohol, or diet. Men have higher levels of stored iron than women [59], and high dietary intake of heme iron has been associated with an increased risk of coronary heart diseases [60]. The potential effect of iron load on the heme oxygenase-1 and bilirubin pathway should not be excluded [61].

The present study has a few strengths. Firstly, the greatest strength for this study was that it quantified the relationship between blood TBIL levels and the risk of IS or AS by conducting linear and nonlinear dose-response analyses. This study demonstrated the protective effect of bilirubin on IS or AS, in particular in men. However, due to the limitations of the meta-analyzed data, the results should be interpreted with caution. Secondly, more than 110 thousand participants from different countries were included in the present meta-analysis and the follow-up period of included studies was as long as 9.4 years. Therefore, the present study had an adequate statistical power to clarify the dose-response relationship between circulating TBIL and the risk of IS/AS and to detect moderate reductions in the risk. Finally, the validated ROBINS-I tool was used to assess the study quality. This is a new tool to evaluate the risk of bias in estimating the comparative effectiveness of interventions from non-randomized studies [26]. It is particularly useful for those undertaking systematic reviews on non-randomized studies. All included studies had adjusted for potential confounding factors, and the methodological assessment was satisfactory.

However, our study also has some limitations. Firstly, available resources for our meta-analysis are insufficient for us to draw an exclusive conclusion. In the present analysis, 4 cross-sectional studies were included to assess the relationship between bilirubin levels and all types of stroke. Though there is a negative relationship between them, the causal relationship could not be confirmed. In addition, only one study on HS was included and our conclusion on the relationship between serum TBIL levels and the risk of HS is not convincing. Secondly, serum TB consists of direct and indirect bilirubin. Few studies have focused on the relationship between different types of bilirubin and stroke. Thirdly, the present study is presented as a meta-analysis by dissecting previously published articles and analyzing their results as a whole. Due to variations and bias in certain respects of the study design, outcome assessment and so on, it is likely that the final conclusion we drew from our analysis is not reflecting the real world result. Therefore, we suggest to confirm our findings with future large-scaled, multi-center, prospective cohort studies. Fourthly, according to a rule of thumb, the validity of a meta-regression with less than 10 studies is limited. Our analysis only included 9 studies. Finally, studies have found that markedly elevated TBIL levels may exert neurotoxic effects under some circumstances $[22,23]$. Thus, results of this analysis should be interpreted with caution in the absence of definitive evidence.

\section{Conclusions}

In conclusion, the present study found a negative dose-response relationship between circulating TBIL and the risk of IS or AS within the physiologic range of serum TBIL in males. Moderately elevated blood TBIL levels might be associated with a diminished incidence of IS. Every $1 \mu \mathrm{mol} / \mathrm{L}$ increment of the blood TBIL level was associated with a $2.2 \%$ decreased risk of IS and a $2.6 \%$ decreased risk of AS. However, due to the limitations in the number of included studies and their quality, large-scaled prospective cohort studies are needed to confirm the conclusion of the current analysis.

\section{Abbreviations}

TBIL: Total bilirubin; DBIL: Direct bilirubin; IBIL: Indirect bilirubin; CVD: Cardiovascular disease; Cl: Confidence interval; HR: Hazard ratio; OR: Odds ratio; RR: Relative risk; RHE: Routine health examination; SCl: Silent cerebral infarction; SIVD: Subcortical ischemic vascular disease; SCOUT: Sibutramine Cardiovascular Outcomes Trial; NFS: Non-fatal stroke; NSHDSC: The Northern Sweden Health and Disease Study; NHANES: The National Health and Nutrition Examination Survey; LI: Lacunar infarction; LA: Leukoaraiosis; IMT: Intima-media thickness; AIS:Acute ischemic stroke; GS: Gilbert's syndrome; LI: Lacunar infarction; LA: Leukoaraiosis

\section{Declarations}

\section{Ethics approval and consent to participate}

This study was exempt from the need for approval by the Ethics Committee of Shanghai Integrated Traditional Chinese and Western Medicine Hospital.

\section{Consent for publication}

Not applicable.

\section{Availability of data and materials}

All data generated or analyzed during this study are included in this article.

\section{Competing interests}

The authors declare that they have no competing interests.

Funding 
This study was supported by the Shanghai Science and Technology Committee Research Projects (17411967700 and 19401935700 awarded to Ping Zhong), Shanghai Hongkou District Health and Family Planning Commission Research Project awarded to Ping Zhong (1802-01) and Shanghai Minhang District Health and Family Planning Commission awarded to Danhong Wu (great discipline of Shanghai Minhang District No.2017MWDXK02).

\section{Authors' contributions}

$\mathrm{XW}$ contributed to study design, literature search, data collection, data analysis, figure preparation, data interpretation, and article writing. YZ contributed to literature search, data collection, data interpretation, and article writing. XY contributed to data collection, data analysis, figure preparation, data interpretation, and article writing. FL contributed to data collection, data interpretation, and article writing. XZ contributed to data collection, data interpretation, and article writing. DW contributed to study design, administrative support, and article writing. PZ contributed to study design, administrative support, and article writing.

\section{Acknowledgements}

We thank Dr Huazheng Liang for proof-reading this manuscript.

\section{References}

1. Benjamin EJ, Blaha MJ, Chiuve SE, et al. Heart disease and stroke Statistics-2017 update: a report from the American heart association. Circulation. 2017;135:e146-

2. Feigin VL. Stroke epidemiology in the developing world. Lancet. 2005;365:2160-1.

3. Wang W, Jiang B, Sun H, et al. Prevalence, incidence, and mortality of stroke in china: Results from a nationwide population-based survey of 480687 adults. Circulation. 2017;135:759-71.

4. Miao Y, Liao JK. Potential serum biomarkers in the pathophysiological processes of stroke. Expert Rev Neurother. 2014;14:173-85.

5. Vitek L. The role of bilirubin in diabetes, metabolic syndrome, and cardiovascular diseases. Front Pharmacol. 2012;3:55.

6. Stocker R, Yamamoto Y, McDonagh AF, et al. Bilirubin is an antioxidant of possible physiological importance. Science. 1987;235:1043-6.

7. Sedlak TW, Saleh M, Higginson DS, et al. Bilirubin and glutathione have complementary antioxidant and cytoprotective roles. Proc Natl Acad Sci USA. 2009;106:5171-6.

8. Syapin PJ. Regulation of haeme oxygenase-1 for treatment of neuroinflammation and brain disorders. Br J Pharmacol. 2008;155:623-40.

9. Kundur AR, Singh I, Bulmer AC. Bilirubin, platelet activation and heart disease: a missing link to cardiovascular protection in Gilbert's syndrome? Atherosclerosis. 2015;239:73-84.

10. Ekblom K, Marklund SL, Johansson L, et al. Bilirubin and UGT1A1*28 Are Not Associated with Lower Risk for Ischemic Stroke in a Prospective Nested Case-Referent Setting. Cerebrovasc Dis. 2010;30:590-6.

11. Kunutsor SK, Bakker SJ, Gansevoort RT, et al. Circulating total bilirubin and risk of incident cardiovascular disease in the general population. Arterioscler Thromb Vasc Biol. 2015;35:716-24.

12. Lee SJ, Jee YH, Jung KJ, et al. Bilirubin and Stroke Risk Using a Mendelian Randomization Design. Stroke. 2017;48:1154-60.

13. Oda E, Kawai R. A possible cross-sectional association of serum total bilirubin with coronary heart disease and stroke in a Japanese health screening population. Heart Vessels. 2012;27:29-36.

14. Jørgensen ME, Torp-Pedersen C, Finer N, et al. Association between serum bilirubin and cardiovascular disease in an overweight high risk population from the SCOUT trial. Nutr Metab Cardiovasc Dis. 2014;24:656-62.

15. Zhou X, Wang L, Liu H, et al. Serum antioxidant levels associated with subcortical ischemic vascular disease. Can J Neurol Sci. 2014;41:375-81.

16. Kim J, Yoon SJ, Woo MH, et al. Differential impact of serum total bilirubin level on cerebral atherosclerosis and cerebral small vessel disease. . 2017;12:e0173736.

17. Schooling CM, Kelvin EA, Jones HE. Alanine transaminase has opposite associations with death from diabetes and ischemic heart disease in NHANES III. Ann Epidemiol. 2012;22:

18. Temme EH, Zhang J, Schouten EG, et al. Serum bilirubin and 10-year mortality risk in a Belgian population. Cancer Causes Control. 2001;12:887-94.

19. Kimm H, Yun JE, Jo J, et al. Low serum bilirubin level as an independent predictor of stroke incidence: a prospective study in Korean men and women. Stroke. 2009;40:3422-7.

20. Perlstein TS, Pande RL, Creager MA, et al. Serum total bilirubin level, prevalent stroke, and stroke outcomes: NHANES 1999-2004. Am J Med. 2008;121:781-8.

21. Zhong P, Wu DH, Ye XF, et al. Association of circulating total bilirubin level with ischemic stroke: a systemic review and meta-analysis of observational evidence. Ann of Transl Med. 2019;7:335.

22. Franchini M, Targher G, Lippi G. Serum bilirubin levels and cardiovascular disease risk: a Janus Bifrons? Adv Clin Chem. 2010;50:47-63.

23. Vitek L, Schwertner HA. The heme catabolic pathway and its protective effects on oxidative stress-mediated diseases. Adv Clin Chem. 2007;43:1-57.

24. Moher D, Liberati A, Tetzlaff J, et al. Preferred reporting items for systematic reviews and meta-analyses: the PRISMA statement. Int J Surg. 2010;8:33641.

25. Stroup DF, Berlin JA, Morton SC, et al. Meta-analysis of observational studies in epidemiology: a proposal for reporting. Meta-analysis Of Observational Studies in Epidemiology (MOOSE) group. JAMA. 2000;283;2008-12.

Page $7 / 11$ 
26. Sterne JA, Hernán MA, Reeves BC, et al. ROBINS-l: a tool for assessing risk of bias in non-randomised studies of interventions. BMJ. 2016;355:i4919.

27. Greenland S, Longnecker MP. Methods for trend estimation from summarized dose-response data, with applications to meta-analysis. Am J Epidemiol. 1992;135:1301-9.

28. Royston P. A strategy for modelling the effect of a continuous covariate in medicine and epidemiology. Stat Med. 2000;19:1831-47.

29. Bagnardi V, Zambon A, Quatto P, et al. Flexible meta-regression functions for modeling aggregate dose-response data, with an application to alcohol and mortality. Am J Epidemiol. 2004;159:1077-86.

30. Egger M, Davey SG, Schneider M, et al. Bias in meta-analysis detected by a simple, graphical test. BMJ. 1997;315:629-34.

31. Lin JP, Vitek L, Schwertner HA. Serum bilirubin and genes controlling bilirubin concentrations as biomarkers for cardiovascular disease. Clin Chem. 2010;56:1535-43.

32. Erdogan D, Gullu H, Yildirim E, et al. Low serum bilirubin levels are independently and inversely related to impaired flow-mediated vasodilation and increased carotid intima-media thickness in both men and women. Atherosclerosis. 2006;184:431-7.

33. Balta S, Balta I, Mikhailidis DP, et al. Bilirubin levels and their association with carotid intima media thickness and high-sensitivity C-reactive protein in patients with psoriasis vulgaris. Am J Clin Dermatol.2014;15:137-42.

34. Kawamoto R, Ninomiya D, Hasegawa Y, et al. Mildly Elevated Serum Bilirubin Levels Are Negatively Associated with Carotid Atherosclerosis among Elderly Persons. PLoS One. 2014;9:e114281.

35. Dullaart RP, Kappelle PJ, de Vries R. Lower carotid intima media thickness is predicted by higher serum bilirubin in both non-diabetic and Type 2 diabetic subjects. Clin Chim Acta.2012;414:161-5.

36. Vitek L, Novotný L, Sperl M, et al. The inverse association of elevated serum bilirubin levels with subclinical carotid atherosclerosis. Cerebrovasc Dis.2006;21:408-14.

37. Wagner $\mathrm{KH}$, Wallner M, Mölzer C, et al. Looking to the horizon: the role of bilirubin in the development and prevention of age-related chronic diseases. Clin Sci (London). 2015;129:1-25.

38. Wang X, Wu DH, Zhong P. Serum bilirubin and ischaemic stroke: a review of literature. Stroke and Vascular Neurology. 2020;0.

39. Yang CW, Liu X, Wang XT, et al. Relationship between serum total bilirubin levels and new cerebral infarction events. Chin J Hypertens. 2014;22:977-80.

40. Lin SP, Lin PY, Jiang HL, et al. Is serum total bilirubin useful to differentiate cardioembolic stroke from other stroke subtypes? Neurol Res. $2015 ; 37: 727-31$.

41. Kurzepa J, Bielewicz J, Stelmasiak Z, et al. Serum bilirubin and uric acid levels as the bad prognostic factors in the ischemic stroke. Int J Neurosci. 2009;119:2243-9.

42. McCarty MF. "latrogenic Gilbert syndrome"--a strategy for reducing vascular and cancer risk by increasing plasma unconjugated bilirubin. Med hypotheses. 2007;69:974-94.

43. Peterson SJ, Frishman WH, Abraham NG. Targeting heme oxygenase: therapeutic implications for diseases of the cardiovascular system. Cardiol Rev. 2009;17:99-111.

44. Targher G. Risk of ischemic stroke and decreased serum bilirubin levels: is there a causal link? Arterioscler Thromb Vasc Biol. 2014;34:702-4.

45. Ali F, Hamdulay SS, Kinderlerer AR, et al. Statin-mediated cytoprotection of human vascular endothelial cells: a role for Kruppel-like factor 2-dependent induction of heme oxygenase-1. J Thromb Haemost. 2007;5:2537-46.

46. Muchova L, Wong RJ, Hsu M, et al. Statin treatment increases formation of carbon monoxide and bilirubin in mice: a novel mechanism of in vivo antioxidant protection. Can J Physiol Pharmacol. 2007;85:800-10.

47. Cui W, Fu G, Wu H, et al. Cadmium-induced heme oxygenase-1 gene expression is associated with the depletion of glutathione in the roots of Medicago sativa. Biometals. 2011;24:93-103.

48. Pomeraniec Y, Grion N, Gadda L, et al. Adrenocorticotropin induces heme oxygenase-1 expression in adrenal cells. J Endocrinol. 2004;180:113-24.

49. Shapiro SM. Defnition of the clinical spectrum of kernicterus and bilirubin-induced neurologic dysfunction (BIND). J Perinatol. 2005;25:54-9.

50. Novotny L, Vitek L. Inverse relationship between serum bilirubin and atherosclerosis in men: a meta-analysis of published studies. Exp Biol Med (Maywood). 2003;228:568-71.

51. Claridge LC, Armstrong MJ, Booth C, et al. Gilbert's syndrome. BMJ. 2011;342:d2293.

52. Schwertner HA, Vítek L. Gilbert syndrome, UGT1A1*28 allele,and cardiovascular disease risk: possible protective effects and therapeutic applications of bilirubin. Atherosclerosis. 2008;198:1-11.

53. Vítek L, Jirsa M, Brodanová M, et al. Gilbert syndrome and ischemic heart disease: a protective effect of elevated bilirubin levels. Atherosclerosis. 2002;160:449-56.

54. Xu T, Zhang J, Xu T, et al. Association of serum bilirubin with stroke severity and clinical outcomes. Can J Neurol Sci. 2013;40:80-4.

55. Pineda S, Bang OY, Saver JL, et al. Association of serum bilirubin with ischemic stroke outcomes. J Stroke Cerebrovasc Dis. 2008;17:147-52.

56. Sagheb Asl E, Taheraghdam A, Rahmani F, et al. Determination of the Predictive Value of Serum Bilirubin in Patients with Ischemic Stroke: A Prospective Descriptive Analytical Study. Adv Pharm Bull. 2018;8:715-9.

57. Jr Adams HP, Bendixen BH, Kappelle LJ, et al. Classification of subtype of acute ischemic stroke. Definitions for use in a multicenter clinical trial. TOAST. Trial of Org 10172 in Acute Stroke Treatment. Stroke. 1993;24:35-41.

58. Li RY, Cao ZG, Zhang JR, et al. Decreased serum bilirubin is associated with silent cerebral infarction. Arterioscler Thromb Vasc Biol. 2014;34:946-51.

59. Sullivan JL. Iron and the sex difference in heart disease risk. Lancet. 1981;1:1293-4.

Page $8 / 11$ 
60. van der A DI, Peeters PH, Grobbee DE, et al. Dietary haem iron and coronary heart disease in women. Eur Heart J. 2005;26:257-62.

61. Kawamura K, Ishikawa K, Wada Y, et al. Bilirubin from heme oxygenase-1 attenuates vascular endothelial activation and dysfunction. Arterioscler Thromb Vasc Biol. 2005;25:155-60.

\section{Tables}

Table 1 Characteristics of included studies examing blood total bilirubin levels and stroke

\section{Figures}

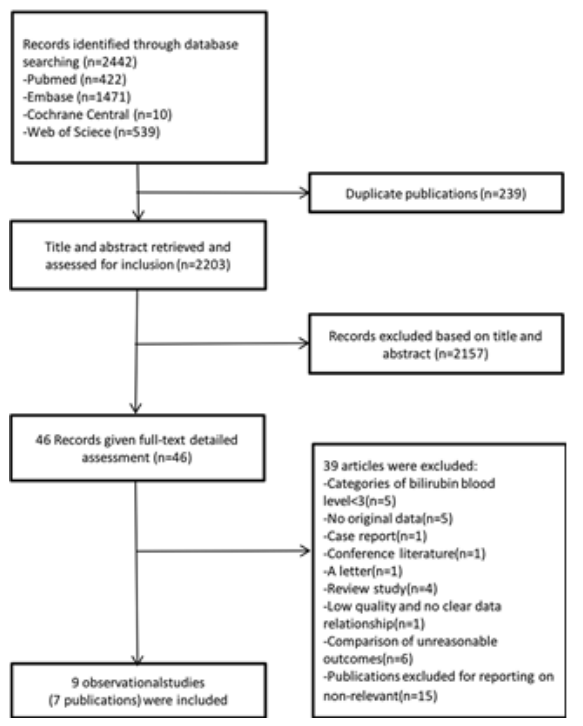

Figure 1

Flow chart of study selection

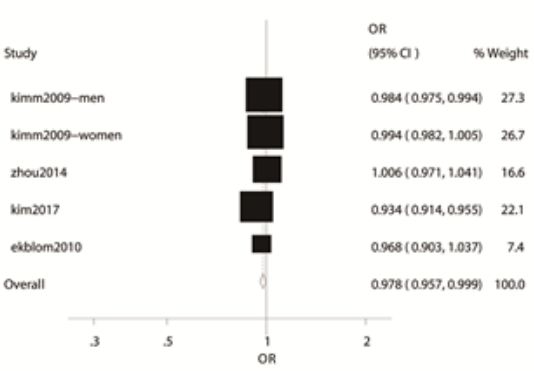

Figure 2

Dose-response analyses between the blood total bilirubin level and the risk of ischemic stroke. The summary OR for ischemic stroke for every $1 \mu$ mol/L increment of the TB level was 0.978 (0.957-0.999).

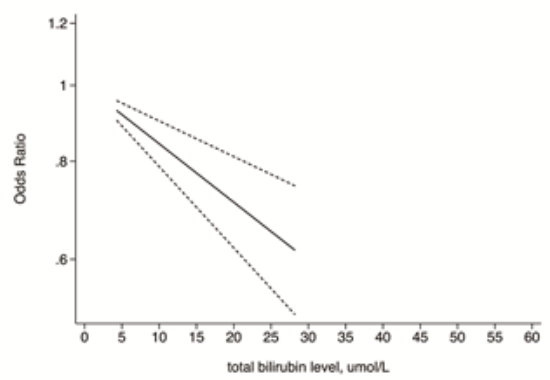

Figure 3

Linear dose-response relationship analysis between bilirubin levels and the risk of ischemic stroke. The solid and short dashed lines represent the estimated OR and its $95 \% \mathrm{Cl}$ (per $1 \mu \mathrm{mol} / \mathrm{L}$ increment). 


\begin{tabular}{|c|c|c|c|c|c|c|c|c|c|c|c|}
\hline $\begin{array}{l}\text { Lead } \\
\text { author, } \\
\text { year of }\end{array}$ & $\begin{array}{l}\text { Name of } \\
\text { study or } \\
\text { source of }\end{array}$ & $\begin{array}{l}\text { Location } \\
\text { of study }\end{array}$ & $\begin{array}{l}\text { Year(s) } \\
\text { of } \\
\text { study }\end{array}$ & $\begin{array}{l}\text { Age } \\
\text { range, } \\
\text { years }\end{array}$ & $\begin{array}{l}\text { Type of } \\
\text { study/follow-up } \\
\text { if prospective }\end{array}$ & $\begin{array}{l}\text { Type } \\
\text { of } \\
\text { stroke }\end{array}$ & $\begin{array}{l}\text { No. } \\
\text { of } \\
\text { cases }\end{array}$ & $\begin{array}{l}\text { Total no. of } \\
\text { participants }\end{array}$ & $\begin{array}{l}\text { Male } \\
\%\end{array}$ & Covariates adjusted for & $\begin{array}{l}\text { Category (Serum total } \\
\text { bilirubin, } \mu \mathrm{mol} / \mathrm{L} \text { ) }\end{array}$ \\
\hline
\end{tabular}
year of source of study years if prospective stroke cases

publication participants

\begin{tabular}{|c|c|c|c|c|c|c|c|c|c|c|c|c|}
\hline \multirow{2}{*}{$\begin{array}{l}\text { Kim } \\
2017[16]\end{array}$} & \multirow[t]{2}{*}{ RHE } & \multirow{2}{*}{$\begin{array}{l}\text { South } \\
\text { Korea }\end{array}$} & \multirow{2}{*}{$\begin{array}{l}2008- \\
2014\end{array}$} & \multirow[t]{2}{*}{$50-79$} & \multirow[t]{2}{*}{ cross-sectional } & \multirow[t]{2}{*}{ SLI } & \multirow[t]{2}{*}{164} & \multirow[t]{2}{*}{1128} & \multirow[t]{2}{*}{$38.1 \%$} & \multirow[b]{2}{*}{$\begin{array}{l}\text { age, sex, hypertension, } \\
\text { diabetes } \\
\text { mellitus, hypercholesterolemia, } \\
\text { current smoking, CAOD, statin } \\
\text { medication, eGFR, hematocrit, } \\
\text { platelet count, albumin, AST, } \\
\text { and triglycerides. }\end{array}$} & \multirow[b]{2}{*}{$\begin{array}{l}\leq 7.7 \mu \mathrm{mol} / \mathrm{L} \\
7.8-11.5 \mu \mathrm{mol} / \mathrm{L} \\
\geq 11.6 \mu \mathrm{mol} / \mathrm{L}\end{array}$} & $\overline{\text { OR }\left(95^{\circ}\right.}$ \\
\hline & & & & & & & & & & & & $\begin{array}{l}\text { Referen } \\
0.92(0.5 \\
1.14(0.2\end{array}$ \\
\hline
\end{tabular}

\begin{tabular}{|c|c|c|c|c|c|c|c|c|c|c|c|c|}
\hline & & & & & & & & & & & & \\
\hline \multirow{4}{*}{$\begin{array}{l}\text { Zhou } \\
2014[15]\end{array}$} & \multirow[t]{4}{*}{ Screening } & \multirow[t]{4}{*}{ China } & \multirow{4}{*}{$\begin{array}{l}2010- \\
2012\end{array}$} & \multirow{4}{*}{$>18$} & \multirow[t]{4}{*}{ cross-sectional } & \multirow[t]{4}{*}{ SIVD } & \multirow[t]{4}{*}{733} & \multirow[t]{4}{*}{1098} & \multirow[t]{4}{*}{$45.7 \%$} & \multirow{4}{*}{$\begin{array}{l}\text { age, gender, BMI, } \\
\text { hypertension, diabetes, } \\
\text { ischemic heart disease, } \\
\text { smoking and alcohol } \\
\text { consumption, HDL, LDL, TG, } \\
\text { TC, FBG, BUN, Cr }\end{array}$} & \multirow{4}{*}{$\begin{array}{l}<9.58 \mu \mathrm{mol} / \mathrm{L} \\
9.58-12.99 \mu \mathrm{mol} / \mathrm{L} \\
>12.99 \mu \mathrm{mol} / \mathrm{L}\end{array}$} & OR $\left(95^{\circ}\right.$ \\
\hline & & & & & & & & & & & & $2.702(1$ \\
\hline & & & & & & & & & & & & $\begin{array}{l}2.702(1 \\
1.729(1\end{array}$ \\
\hline & & & & & & & & & & & & Referen \\
\hline
\end{tabular}

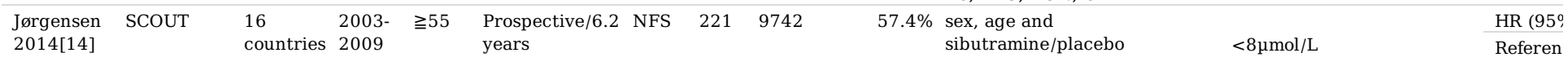

sibutramine/placebo $\quad<8 \mu \mathrm{mol} / \mathrm{L} \quad$ Referen

$10-13 \mu \mathrm{mol} / \mathrm{L} \quad 0.819(0$

$>13 \mu \mathrm{mol} / \mathrm{L} \quad 0.803(0$

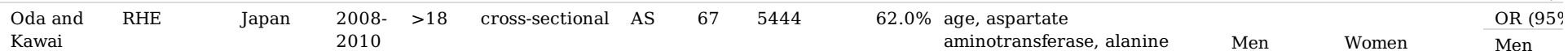

$\begin{array}{llll}\text { aminotransferase, alanine } & \text { Men } & \text { Women } \\ \text { aminotransferase, gamma } & & 0.9-9.3 \mu m o l / L\end{array}$

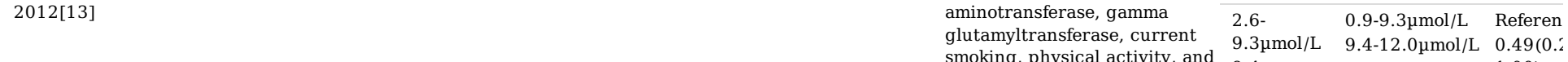
$\begin{array}{llll}\text { smoking, physical activity, and } & 9.3 \mu \mathrm{mol} / \mathrm{L} & 9.4-12.0 \mu \mathrm{mol} / \mathrm{L} & 0.49(0.2 \\ \text { everyday drinking } & 12.1- & 1.00)\end{array}$

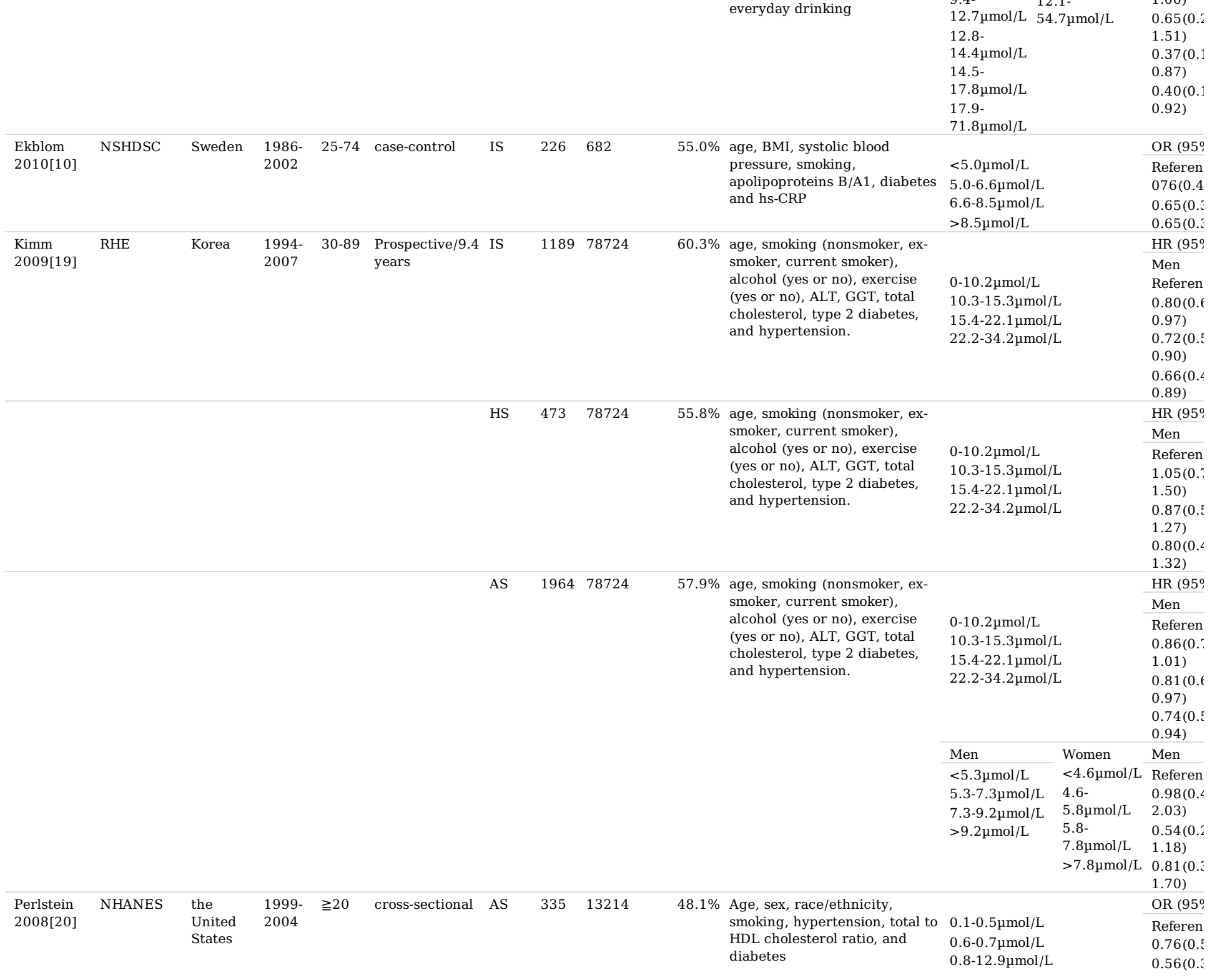

Per 1.71 $\mu \mathrm{mol} / \mathrm{L}$ increase in $\quad 0.91(0 . \varepsilon$ total bilirubin 


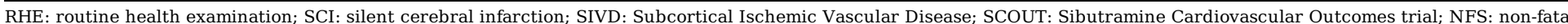

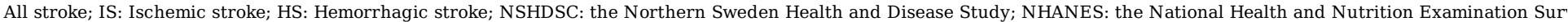

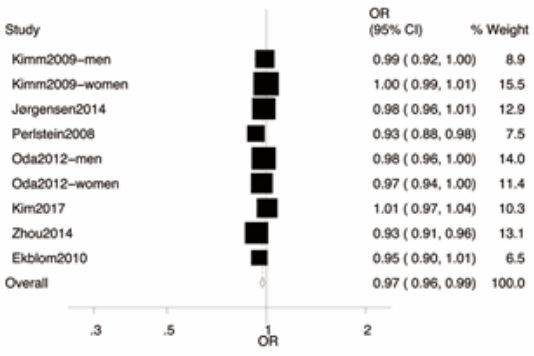

\section{Figure 4}

Dose-response analyses between the blood total bilirubin level and the risk of stroke. The summary OR for stroke for every $1 \mu \mathrm{mol} / \mathrm{L}$ increment in the TB level was $0.974(0.956-0.992)$.

\section{Supplementary Files}

This is a list of supplementary files associated with this preprint. Click to download.

- Supplementarymaterial.pdf 